\title{
RESULTADOS PRELIMINARES DEL CRECIMIENTO DE NEONATOS DE LAGARTO NEGRO Melanosuchus niger (Spix 1825), (Crocodylia, Alligatoridae), NACIDOS EN CAUTIVERIO EN IQUITOS - PERU
}

Luis A. SICCHAR ${ }^{1,2}$, Arturo ACOSTA ${ }^{1,2}$, Martha RENGIFO ${ }^{1,2}$, Maria BOSANTES ${ }^{1}$, Jonny HIDALGO ${ }^{1}$, Tomy VILLACORTA ${ }^{1}$., Marcelino PANDURO ${ }^{1}$, Susana RAMÍREZ ${ }^{1}$, Maximiliano MONGE ${ }^{1}$, Meuri YAHUARCANI ${ }^{1}$, Cyntia SÁNCHEZ ${ }^{1}$, Gisela SOUSA ${ }^{1}$, Claudia SICCHAR $^{1}$

1 Universidad Nacional de la Amazonía Peruana-Facultad de Ciencias Biológica. Pevas cuatra 4, Iquitos, Perú.

2 Universidad Nacional de la Amazonía Peruana - Facultad de Ciencias Biológica., Proyecto Cría y Reproducción de Caimanes Amazónicos en Cautiverio. E-mail: lasv_bb@hotmail.com

\section{RESUMEN}

Se reporta la crianza en cautiverio de neonatos de Melanosuchus niger, a partir de la incubación de huevos de tres nidos recolectados de áreas naturales por el sistema de rancheo. Sobrevivieron 06 neonatos que fueron colocados en una pecera de vidrio de $1.40,0.40 \times 0.50 \mathrm{~m}^{3}$, durante nueve meses. Un neonato murió por ataque de roedores y dos por prematuridad. Luego fueron trasladados por tres meses en un ambiente semi-natural, de $8 \times 12 \mathrm{~m}^{2}$, donde compartieron espacio y alimentación con las otras tres especies de caimanes amazónicos, todos juveniles. La alimentación fue a base de peces. Los neonatos, al momento de la eclosión, tuvieron una longitud total de $29.93 \mathrm{~cm}=299.3 \mathrm{~mm}(29.6 \mathrm{a} 30.8 \mathrm{~cm})$, y un peso de $86.67 \mathrm{~g}(82 \mathrm{a} 93 \mathrm{~g})$. En doce meses tuvieron un crecimiento promedio en longitud total de $46.22 \mathrm{~cm}=462.2 \mathrm{~mm}$ y peso de $286.14 \mathrm{~g}$. El crecimiento mensual de la longitud total tuvo una ganancia promedio de $3.01 \mathrm{~cm}=30.1 \mathrm{~mm}$, con crecimiento diario de $0.12 \mathrm{~cm}=1.2 \mathrm{~mm}$; y el incremento mensual de peso de $55 \mathrm{~g}$, con un incremento diario de $1.83 \mathrm{~g}$.

PALABRAS CLAVE: Melanosuchus niger, crianza, cautiverio.

\section{PRELIMINARY RESULTS OF GROWTH OF INFANTS BLACK CAIMAN Melanosuchus niger (Spix 1825) (Crocodylia, Alligatoridae), BORN IN CAPTIVITY IN IQUITOS - PERU}

\begin{abstract}
We report on the rearing of captive hatchlings of Melanosuchus niger, incubated from eggs collected from three nests in a wildlife rescue area. Six hatchlings survived and were placed in a glass container $(1.4 \mathrm{~m} 0.4 \mathrm{~m}$ $\mathrm{x} 0.5 \mathrm{~m}^{3}$ ) for nine months. One hatchling was killed by rodents, two others died due to premature hatching. After nine months the animals were transferred to a semi-natural enclosure $\left(8 \mathrm{~m} \mathrm{x} 12 \mathrm{~m}^{2}\right)$ which they shared with juveniles from the other three species of Amazonian caimans. The animals were fed fish. At hatching, the neonates had a total length of $29.93 \mathrm{~cm}$ (range: 29.6-30.8 cm) and a body mass of $86.67 \mathrm{~g}$ (range: 82-93 g). After 12 months, mean length and body mass gain were $46.22 \mathrm{~cm}$ and $286.14 \mathrm{~g}$, respectively. Monthly growth rates were $3.01 \mathrm{~cm}$, daily growth rates $0.12 \mathrm{~cm}$; monthly body mass gain was $55 \mathrm{~g}$, daily body mass gain $1.83 \mathrm{~g}$.
\end{abstract}

KEYWORDS: Melanosuchus niger, breeding, captive. 


\section{INTRODUCCIÓN}

La tasa de crecimiento es un parámetro poblacional importante en los cocodrilianos. Varios factores demográficos dependen de la rapidez con la que crecen los individuos. Por ejemplo, el crecimiento juega un papel importante en la sobrevivencia de las crías, ya que entre más rápido crecen, existen menos probabilidades de que sean depredadas (Jacobsen \& Kushlan, 1989). Por su parte, Thorbjarnarson (1989), reporta que la tasa de crecimiento en los Crocodylia en general, disminuye con la edad; se da una mayor tasa de crecimiento durante los primeros 3 meses de vida, en ocasiones excediendo los $0.3 \mathrm{~cm}$ de longitud total/día, y disminuye a partir del primero o segundo año. En la mayoría de las poblaciones de cocodrilos, el crecimiento es considerablemente mayor en los neonatos que en juveniles y adultos.

Actualmente existen cinco especies de cocodrilos, de las cuales, uno pertenece a la familia Crocodilidae Crocodilus acutus (Cuvier, 1807) "cocodrilo de tumbes", de distribución muy limitada al noroeste del Perú y categorizado "En Peligro Crítico" (Ministerio de Agricultura - Decreto Supremo $\mathrm{N}^{\circ}$ 004-2014-MINAGRI), y las otras cuatro especies pertenecen a la familia Alligatoridae y se encuentran distribuidos en la Amazonía peruana, como Melanosuchus niger (Spix, 1825), “caimán o lagarto negro" categorizado como "casi amenazado" (NT) en el Decreto Supremo ${ }^{\mathrm{o}}$ 004-2014-MINAGRI; Caiman crocodilus (Linnaeus, 1758), "caimán o lagarto blanco, challua lagarto", no está categorizado en él, D.S. N $N^{\circ} 004-$ 2014-MINAGRI, "ni en peligro, ni vulnerable, ni casi amenazado"; Paleosuchus trigonatus (Schneider, 1801), "dirin dirin, lagarto enano, lagarto del aguajal, bola-bola" categorizado como “casi amenazado" (NT); y Paleosuchus palpebrosus (Cuvier,1807), "dirin dirin, lagarto enano, lagarto del aguajal, bola-bola" categorizado "en peligro" (EN) en el mencionado decreto supremo. Según UICN Tejado (2012), M. niger es considerado LR/cd (Riesgo bajo/dependiente de conservación, en función de cada país) y en la CITES figura en el apéndice I, Cites (2015). Su aprovechamiento no sostenible ha provocado que esta especie se encuentre en peligro de extinción, según Plotkin et al (1983) y Thorbjarnarson (2010).
Trabajos relacionados con la biología reproductiva en áreas naturales de $M$. niger en la zona colombiana, son citados en Brieva (2002) y Neira (1994), quienes abordan también aspectos sobre medición de neonatos. En Ecuador, Villamarín (2006), hace referencia sobre anidación. En Brasil, Rebêlo \& Magnusson (1983) y Da Silveira et al (2013), Dowling \& Brazaitis (1966) y Magnusson \& Sanaiotti (1995), abordan aspectos relacionados a su crecimiento en áreas naturales y hábitats amazónicos de ese país; así como sobre crecimiento en cautiverio. Sobre reproducción en Perú, existe el informe periodístico de esta especie (Cisneros, 2012). La mayor cantidad de reportes están direccionados a su ecología alimentaria (Herron et al., 1990; Horna et al., 2003; Herron, 1991; De la Ossa et al., 2010), abundancia; Tejado (2012), taxonomía (Rueda - Almonacid et al., 2007; Thorbjarnarson, 2010); estado embrionario (Vieira etal., 2011).

Existen experiencias de manejo con otras especies de cocodrilianos. En Crocodylus acutus 24 meses de crecimiento con la alimentación de cinco días a la semana (Rodríguez \& Rodríguez, 1989, 1991); sobre tratamientos en densidad, dieta y frecuencia de neonatos (Piedra et al., 1996-1997); en crecimiento promedio de longitud y peso, en clase y edades, y promedios de crecimiento de crías de diferentes edades con dietas patrones y experimentales (Pérez \& Escobedo-Galván, 2007; Meraz et al., 2008; Cupul-Magaña et al., 2004a; Cupul-Magaña et al., 2004b; Pérez Gómez et al., 2009), encontrándose que las tasas de crecimiento en longitud y peso varían mucho entre los individuos jóvenes, sub-adultos y adultos (Charruau et al., 2010). En Crocodylus intermedius, evaluaron la influencia de la temperatura del aire y del agua en el crecimiento en condiciones de cautiverio (Pérez \& Rodríguez, 2005; Pérez, 2007). En Crocodylus moreletii sobre el crecimiento en cautiverio de (Barrios-Quiroz \& Casas-Andreu, 2010). En Caiman crocodilus crocodilus (Staton \& Dixon, 1977); Caiman crocodilus fuscus (Rodríguez, 1988 y 1989; Pérez, 2000 y 2001; Jiménez-Alonso et al., 2006).

El presente trabajo tuvo como objetivo generar información sobre el crecimiento que presentan los neonatos del lagarto negro Melanosuchus niger nacidos y criados en cautiverio en la ciudad de Iquitos - Perú 


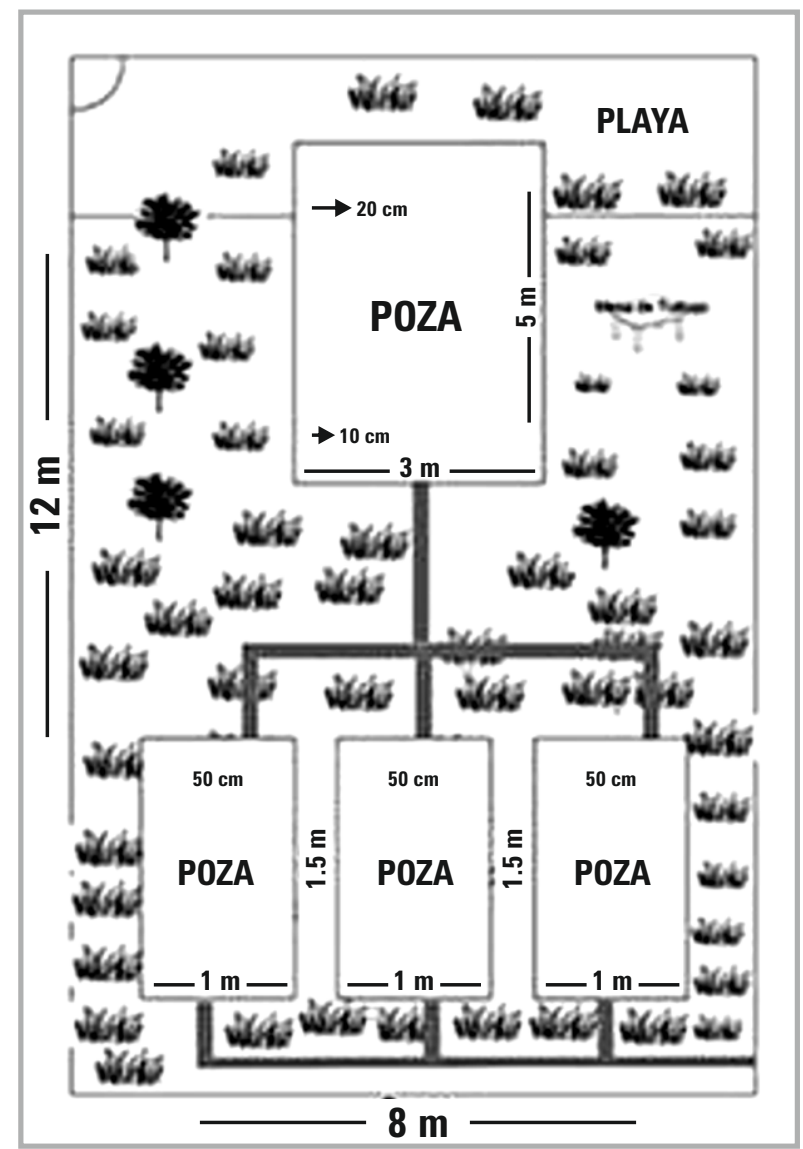

Figura 1. Representación esquemática del ambiente semi - natural donde estuvieron alojados los neonatos de caimanes negros con las otras tres especies de caimanes amazónicos.

\section{MATERIALES Y MÉTODOS}

Esta investigación se desarrolló entre los meses de noviembre 2010 - noviembre 2011, en el Centro de Rescate de Caimanes Amazónicos de la Universidad Nacional de la Amazonía Peruana Facultad de Ciencias Biológicas de la ciudad de Iquitos - Perú. El periodo de evaluación fue de 12 meses.

\section{Incubación y marcaje de neonatos}

Se trabajó con 6 neonatos de “caimán negro", de un total de 9 nacidos en cautiverio entre el 08 y 12 de noviembre del 2010, por medio de la incubación de huevos de tres nidos recolectados de áreas naturales por el sistema de rancheo. Los neonatos eclosionaron en horas de la madrugada y fueron marcados haciendo un corte en las escamas caudales para el reconocimiento individual, donde el caimán negro $\mathrm{N}^{\circ} 2$ fue macho y el resto hembras.

\section{Crianza en cautiverio}

La cría de los caimanes negros constó de dos etapas: la primera en una pecera de vidrio de 1.40 $0.40 \times 0.50 \mathrm{~m}^{3}$, por nueve meses y la segunda en un ambiente semi-natural de $8 \times 12 \mathrm{~m}^{2}$ por tres meses, con piso de tierra de tipo arcilla- arenosa, donde se construyeron pozas (para el mantenimiento de peces que sirvieron de alimento a los caimanes), rodeadas de vegetación arbustiva, hierbas y árboles, y con cerco perimétrico de madera para evitar el escape de los ejemplares (Figura 1). Los neonatos de lagarto negro estuvieron conviviendo con las otras tres especies de caimanes amazónicos, todos juveniles.

\section{Alimentación}

La alimentación de los neonatos en los dos ambientes, fue con peces forrajeros como Ctenobrycon hauxwellianus "mojarra" y Trichogaster sp. "gurami", obtenidos de su medio 
natural y consumidos en forma "ad libitum". Hasta los 3 meses se les proporcionó "mojarras"; el primer mes en número de 80 peces semanalmente; en el segundo mes 150 peces semanalmente; en el tercer mes un promedio de 300 peces semanales; a partir del cuarto mes con "guramis", en número de 250 peces en forma semanal. En el medio semi - natural (noveno al décimo segundo mes), se suministraron 800 peces de esta especie cada quince días.

La biomasa semanal de las "mojarras" en el primer mes fue de $200 \mathrm{~g}$ (longitud total de 2.4 a 2.8 cm y $2.5 \mathrm{~g}$ de peso) y a partir del segundo mes fue de $450 \mathrm{~g}$ (longitud total de $3.33 \mathrm{~cm}$ y $3 \mathrm{~g}$ de peso). En el tercer mes la biomasa semanal fue de $900 \mathrm{~g}$. A partir del cuarto al octavo mes de vida, en los ambientes de la pecera, la alimentación fue cambiada a "guramis", con una biomasa semanal de $870 \mathrm{~g}$ (longitud total promedio de $4.89 \mathrm{~cm}$ y $3.48 \mathrm{~g}$ de peso) y en el medio semi-natural la biomasa de "guramis" fue de 2,784 g cada 15 días.

\section{Registro de longitud y peso}

La longitud total fue determinada utilizando un vernier digital (0.01 $\mathrm{mm}$ de precisión), sauriómetro, (ictiómetro de mayor tamaño), con precisión de 1 $\mathrm{mm}$ y el peso con balanza digital $(0.001 \mathrm{~g}$ de precisión).

El crecimiento se determinó por Crecimiento $=$ Longitud final - Longitud inicial. La ganancia de peso Ganancia de Peso = Peso Actual - Peso Anterior. La biomasa de los peces otorgados, se calculó multiplicando el peso promedio de la especie de pez por el número de organismos vivos otorgados.

\section{RESULTADOS Y DISCUSIÓN}

\section{Tiempo de incubación, biometría de huevos y eclosión}

Se recolectaron tres nidos procedentes de áreas naturales, por el sistema de rancheo, uno de $29 \mathrm{y}$ dos de 33 huevos. El tiempo de incubación tuvo un promedio de 128 días. Las dimensiones de los huevos están expresadas en la Tabla 1. Del primer nido ( $\mathrm{n}=29$ huevos) eclosionaron 3 ejemplares ( 1 neonato logrado, murió por ataque de ratas Rattus rattus y 25 no eclosionaron); del segundo nido ( $\mathrm{n}=$ 33 huevos), nacieron 6 neonatos (02 murieron por prematuridad por presentar el saco vitelino muy

Tabla 1. Biometría de huevos de Melanosuchus niger obtenidas en el estudio.

\begin{tabular}{ccccc}
\hline Nido & $\mathbf{N}^{\circ}$ de huevos & $\begin{array}{c}\text { Peso promedio } \\
(\mathbf{g})\end{array}$ & $\begin{array}{c}\text { Ancho promedio } \\
(\mathbf{m m})\end{array}$ & $\begin{array}{c}\text { Largo promedio } \\
(\mathbf{m m})\end{array}$ \\
\hline 1 & 29 & 82,63 & 49,63 & 120,31 \\
2 & 33 & 82,99 & 49,64 & 121,25 \\
$3^{*}$ & 33 & 82,30 & 49,62 & 119,79 \\
\hline
\end{tabular}

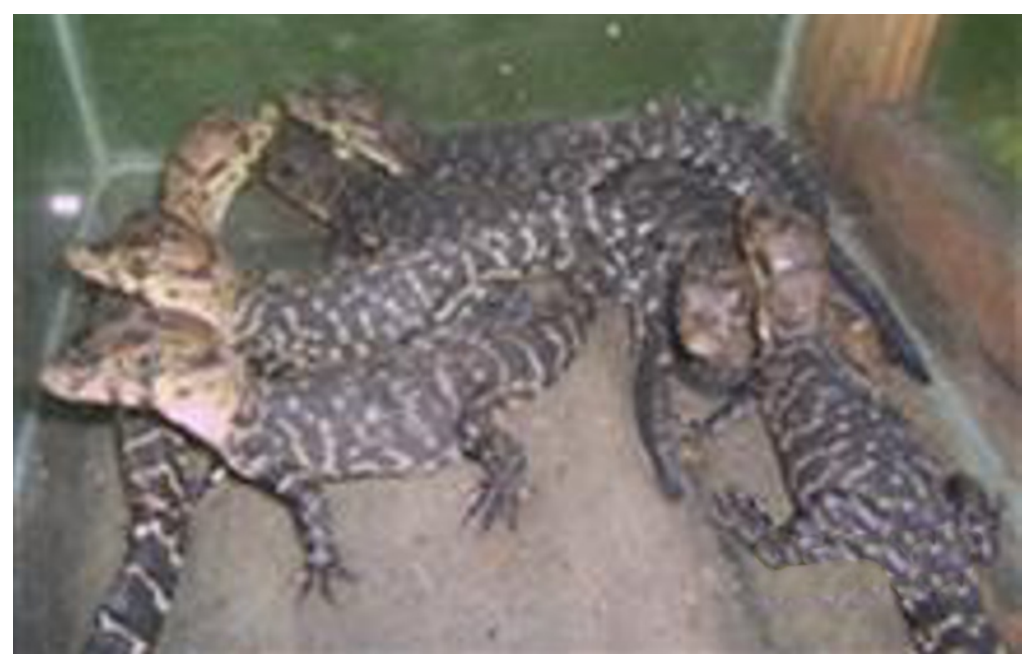

Figura 2. Neonatos de Melanosuchus niger a 24 horas de eclosión. (Foto L. Sicchar (C) UNAP). 
abultado produciéndose infección de la misma y 25 huevos no eclosionaron) y del tercer nido $(\mathrm{n}=33$ huevos) todos murieron en la primera etapa de su desarrollo en el estadio 4 de 10 días de desarrollo embrionario, caracterizado por presentar 12 pares de anillos que son visibles sobre el dorso del embrión (Vieira et al, 2011), probablemente, por haberse tratado de huevos colocados por la madre en vegetación flotante (tamalones) y muy próximos al agua. El resultando total fue de 06 neonatos vivos (Figura 2). Los neonatos sobrevivientes estuvieron compuestos por un macho y cinco hembras, marcados según la metodología de Rueda et al. (2007), y colocados en una pecera de vidrio (Figura 2) para la reabsorción completa del saco vitelino y cicatrización del ombligo (Figura 3). La tasa de sobrevivencia en el crecimiento de Melanosuchus niger en esta investigación fue de $100 \%$.

\section{Crecimiento en longitud y ganancia de peso}

Los seis neonatos, al momento de nacer, tuvieron una longitud de 29.6 a $30.8 \mathrm{~cm}$ (promedio de 29.93 $\mathrm{cm}=299.3 \mathrm{~mm}$ ) y pesaron de $82 \mathrm{a} 93 \mathrm{~g}$ (promedio de $86.67 \mathrm{~g}$ ). Los caimanes negros, durante los doce meses de observación, tuvieron un crecimiento promedio en longitud de $46.22 \mathrm{~cm}=462.2 \mathrm{~mm}$ y peso de $286.14 \mathrm{~g}$; con un incremento mensual longitud de $3.01 \mathrm{~cm}=30.1 \mathrm{~mm}$ y peso de $55 \mathrm{~g}$ y el incremento diario de longitud fue $0.12 \mathrm{~cm}=1.2$ $\mathrm{mm} /$ día; el peso indicó $1.83 \mathrm{~g}$. Individualmente, el incremento en longitud y peso mensual de los neonatos de los caimanes fue variable. Los incrementos de longitud y peso por mes y por animal están indicados en las Tablas 2 y 3.

El incremento notable de longitud y peso de los caimanes neonatos se observó en el octavo mes de crianza y la expresión más elevada se evidenció en el onceavo mes, cuyas longitudes marcaron de 2 a $8.6 \mathrm{~cm}$ de crecimiento y el peso de 50 a $300 \mathrm{~g}$, no obstante, de compartir el ambiente con 14 ejemplares juveniles de Caiman crocodilus, 05 de Paleosuchus trigonatus y 02 Paleosuchus palpebrosus. El comportamiento observado en los neonatos de Melanosuchus niger, fue de individuos tranquilos, no agresivos con las otras especies de caimanes y que toleraban bien la presencia del hombre. Muchas veces fueron observados formando un solo grupo, amontonados y alejados de los otros caimanes cuando se asoleaban, pero en la mayoría de casos permanecieron juntos con las demás especies.

Los trabajos sobre Melanosuchus niger, en la Amazonía peruana, están principalmente referidos a su abundancia, selección de hábitat y bioecología, donde destacan los trabajos de Herron et al. (1990), Tejado (2012) y el informe periodístico sobre reproducción de Cisneros (2012). Herron, (1991),

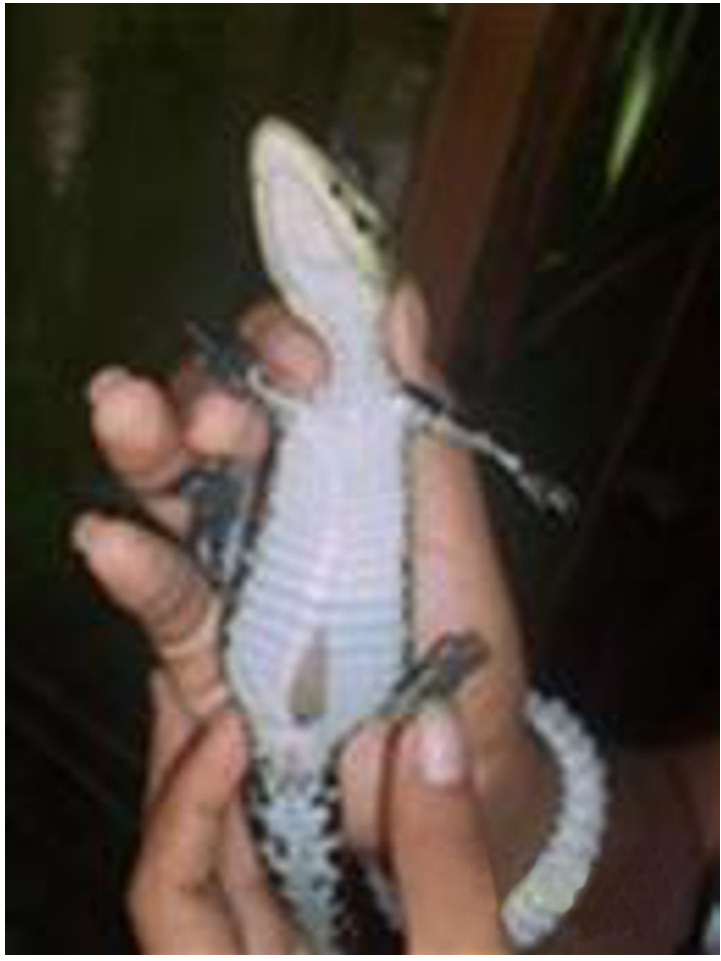

Figura 3. Melanosuchus niger mostrando la reabsorción del saco vitelino a 24 horas de su eclosión. (Foto L. Sicchar (c) UNAP)

afirma haber observado tres crías moviéndose hacia el agua, capturando un neonato antes de abandonar el nido, con medidas de $30.7 \mathrm{~cm}$ de longitud total y un peso de $91 \mathrm{~g}$, coincidentes con los resultados del presente estudio, cuyas longitudes variaron de 29.6 a $30.8 \mathrm{~cm}$ (promedio de $29.93 \mathrm{~cm}$ ) y peso de $82 \mathrm{a} 93 \mathrm{~g}$ (promedio de $86.67 \mathrm{~g}$ ); los autores no reportan datos de crecimiento.

Neira (1994), reporta que obtuvo datos de observación y medición sobre la reproducción en cautiverio del caimán negro (Melanosuchus niger), tomados durante tres años consecutivos sobre una misma hembra, periodos de postura, incubación, número de huevos y su morfometría. En este último aspecto, refiere la biometría de 08 neonatos cuyas medidas variaron de 29.5 a $31.6 \mathrm{~cm}$ (promedio de $30.4 \mathrm{~cm}$ ), pero no reporta el peso de las crías. Nuestros neonatos al nacer tuvieron una longitud de 29.6 a $30.8 \mathrm{~cm}$, con un promedio de $29.93 \mathrm{~cm}$, y un peso de 82 a $93 \mathrm{~g}$, con un promedio de $86.67 \mathrm{~g}$, que indican que nuestros neonatos tuvieron $0.47 \mathrm{~cm}$ debajo de los valores reportados por este autor, y que algunos neonatos (05 especímenes) fueron aislados durante 20 días por pérdida de peso que varió de 5.3 a $9.3 \mathrm{~g}$ (promedio de $7.4 \mathrm{~g}$ ), muriendo uno de ellos a los 15 días. Nuestros neonatos tuvieron un 
crecimiento constante (Tablas 2 y 3 ), sin presentarse problemas de salud (Figuras 2 y 3 ). Considera que ésta pérdida corresponde a la absorción del vitelo, agregando que está por identificar el tiempo que sostiene a los neonatos durante la absorción del saco vitelino; en nuestros caimanes negros, se aplicó experiencias de manejo de neonatos reportados en otras especies de cocodrilianos.

Larriera et al. (2005), menciona que en Ecuador el programa de ranching de Melanosuchus niger carece de un Programa Nacional para la conservación de cocodrilos y existe específicamente un criadero de esta especie, en la ciudad de Coca en la Amazonía ecuatoriana. Este criadero cuenta con instalaciones para aproximadamente unos 300 ejemplares, y en esa fecha existió un stock de 48 caimanes. Los principales problemas fueron, diferentes enfermedades que provocaron la muerte de aproximadamente 100 ejemplares producto de la mala conservación de los alimentos suministrados e indican que tienen problemas en la obtención de neonatos en vida silvestre. No se reporta el nacimiento de neonatos de Melanosuchus niger.

Villamarín (2006), reporta sus experiencias en el rango de dimensiones de los huevos de Melanosuchus niger usados en su investigación, fluctuaron entre $76.12-97.59 \mathrm{~mm}$ de largo, $42.65-$ $51.45 \mathrm{~mm}$ de ancho y $90-155 \mathrm{~g}$ de peso. Nuestros datos tuvieron un promedio de $119.79-121.25 \mathrm{~mm}$ de largo, 49.62 - $49.64 \mathrm{~mm}$ de ancho y $82.30-$ 82.99 g de peso, observando que los huevos de nuestros "caimanes negros" fueron más largos y anchos, pero relativamente con un peso menor.

Albarracin \& Madera (2011), mencionan que encontraron 04 cuatro grupos de neonatos con 41 individuos de Melanosuchus niger, en la laguna de Limoncocha (Ecuador) e identificaron a la madre de cada tropa en cuidado parental en una noche, sin hacer referencia a longitudes y peso que tenían los neonatos observados y seguimiento de estos durante un período de sus vidas.

Dowling \& Brazaitis (1966), reportó para Melanosuchus niger un crecimiento de $0.62 \mathrm{~mm} /$ día en cautiverio. Nuestros datos indican $0.12 \mathrm{~cm} /$ día $=$ $1.2 \mathrm{~mm} /$ día cantidad mayor a lo reportado por estos autores, sin especificar el tipo de alimentación otorgado en esa investigación.

Rebêlo \& Magnusson (1983), estimaron que el caimán negro crece de $30-35 \mathrm{~cm}$ por año. Señalan que estas cifras implican que después de entrar a $100 \mathrm{~cm}$, que es el grupo más intensamente cazado requiere de aproximadamente tres años de crecimiento para llegar a la madurez sexual. Nuestra investigación expresó un crecimiento anual de $46.22 \mathrm{~cm}$, cantidad superior a lo reportado por el autor, probablemente a que Melanosuchus niger en áreas naturales tienen que buscar sus alimentos en diferentes lugares, si es que lo encuentran y competir con otros animales que tienen régimen alimenticio muy similar.

Magnusson \& Sanaiotti (1995), para Melanosuchus niger criados en cautiverio, determinaron un crecimiento de $0.62 \mathrm{~mm} /$ día y un crecimiento constante de $0.93 \mathrm{~mm} /$ día, sin mencionar el tipo de alimentación, cantidad menor a los resultados de esta investigación que reporto $1.2 \mathrm{~mm} /$ día.

Cisneros (2012), nombrando a la Blga. Gabyluz Vela, informa que esta investigadora recolectó 30 huevos de "lagarto negro" transportados en su misma hojarasca para la reanidación. Los huevos eclosionaron a las doce semanas de incubación, naciendo 29 lagartitos, 24 fueron hembras y 5 machos. Fueron liberados con 2 meses de edad con 100 gramos de peso y 36 centímetros de longitud promedio. Este reporte no menciona las medidas de los neonatos al momento de nacer, ni el tipo de alimentación, ni el lugar donde estuvieron alojados antes de sus liberaciones durante los dos meses que estuvieron en su poder. Nuestros neonatos, en dos meses, alcanzaron un promedio de $111.66 \mathrm{~g}$ de peso y una longitud de $36.48 \mathrm{~cm}$.

Da Silveira et al. (2013), reportan tasas de crecimiento de Melanosuchus niger en áreas naturales en machos que variaron desde 2.7 hasta $16.4 \mathrm{~cm} /$ año y en hembras desde 1.9 hasta $12.5 \mathrm{~cm} /$ año, cantidades menores a las obtenidas en esta investigación, que fueron de $46.22 \mathrm{~cm} /$ año. El trabajo de estos autores no reporta si se trata de neonatos, pero si en individuos que tuvieron medidas comprendidas entre 1.0 a 4.4 años, probablemente sub-adultos y adultos.

Esta investigación coincide a lo reportado por Thorbjarnarson (1989), porque nuestros datos se encuentran dentro de estos parámetros y sólo en dos casos $(0.5,0.87 \mathrm{~cm}$ de longitud total $/$ día $)$ superan a lo reportado por el autor, que no menciona tasas de incremento en pesos de los cocodrilianos, que en este trabajo siempre fue creciente. En su mejor proceso representó $10 \mathrm{~g}$ /día de incremento en peso, no obstante, de tratarse de una monodieta consistente en peces que ellos capturaban adlibitum.

En el manejo de otra especie de cocodriliano, Rodríguez \& Rodríguez (1989) indican que 84 neonatos de Crocodylus acutus tuvieron una longitud al nacer de $307 \mathrm{~mm}$ (promedio de $246 \mathrm{~mm}$ ) y al cabo de 12 meses de observación en crecimiento obtuvieron una longitud de $48.18 \mathrm{~cm}$., y un peso de 390.3 g. Nuestra investigación reporta que $M$. niger al momento de nacer es más grande en $53.3 \mathrm{~mm}$ que C. acutus pero después de doce meses de crecimiento en longitud nuestros datos son menores 


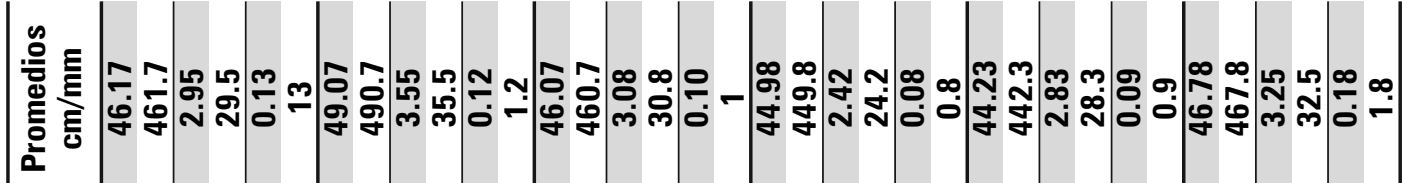

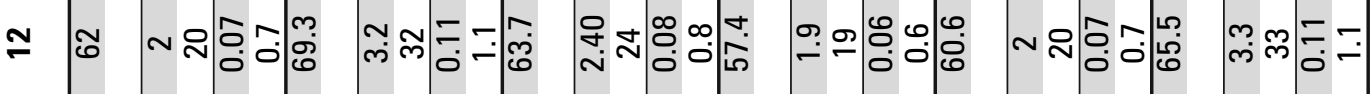

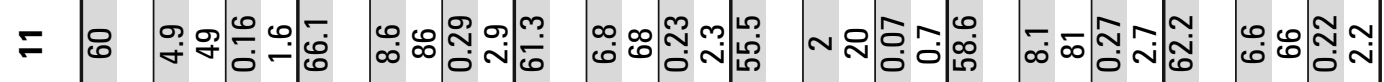

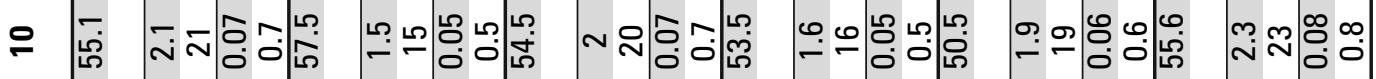
* D

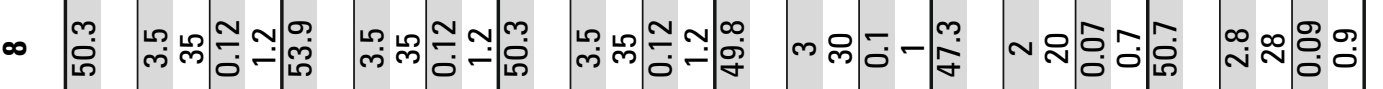

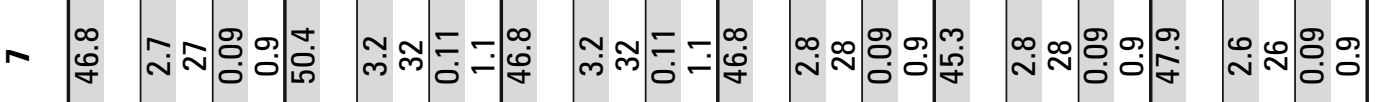

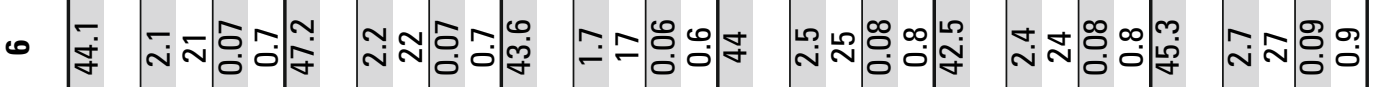
L

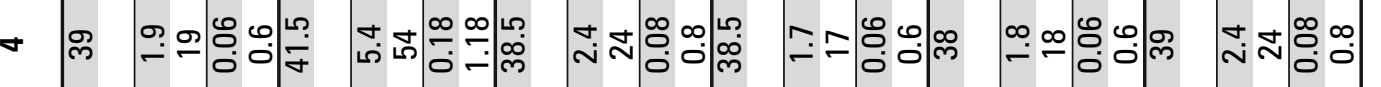

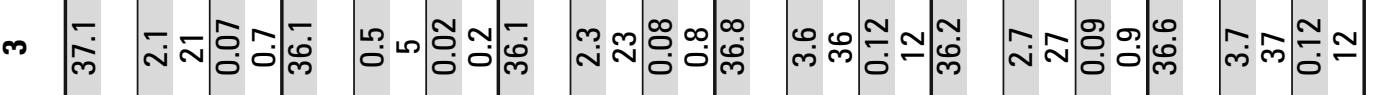

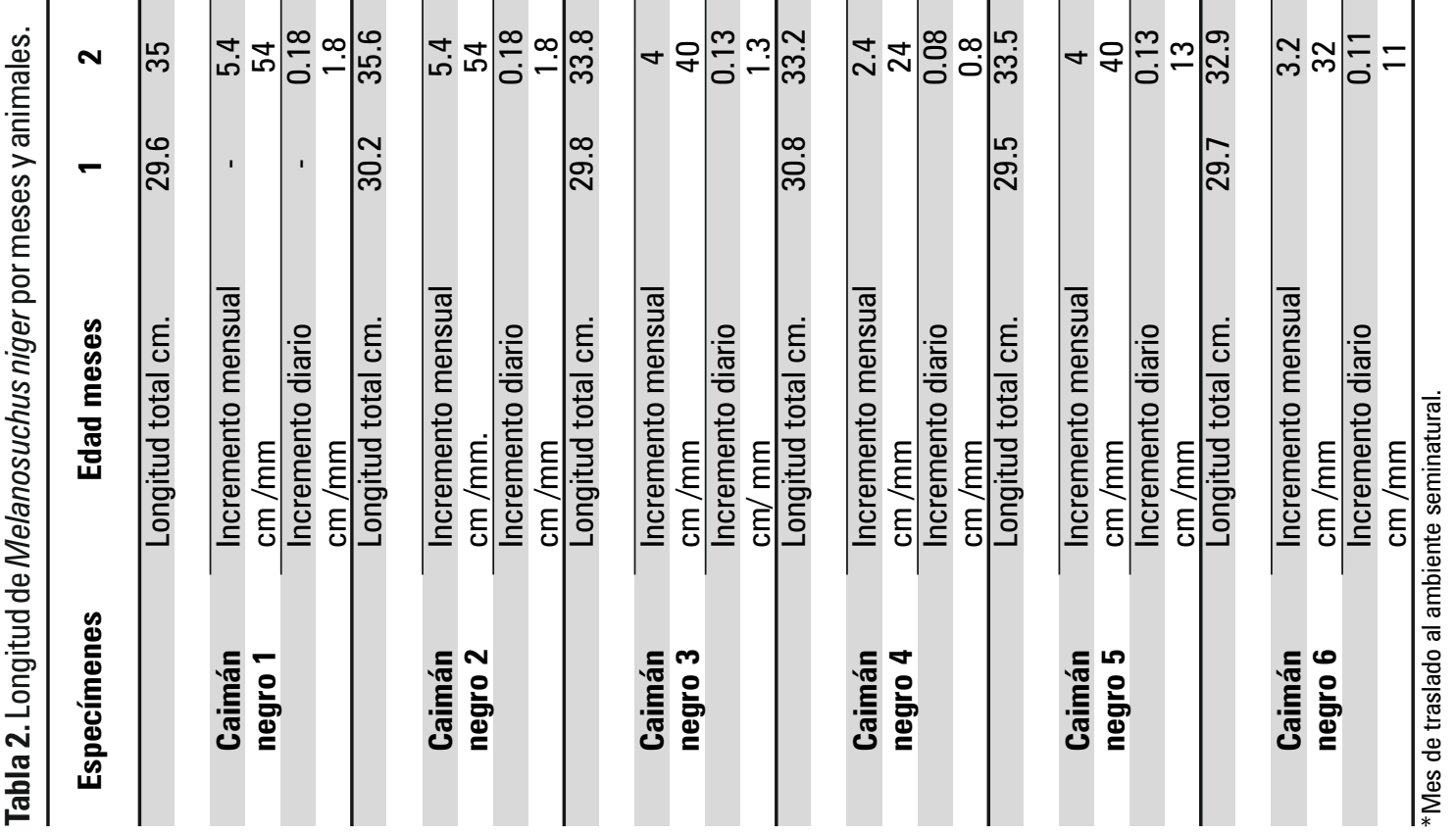


en $1.99 \mathrm{~cm}$ en longitud, $\mathrm{y}$ en peso $104.16 \mathrm{~g}$ debido a ser especies diferentes de cocodrilos y que la alimentación de estos cocodrilos se incluyó el suministro de una dieta variada complementada con vitaminas, glucosa y un antibiótico (Oxitetraciclina veterinaria). Nuestro caso fue exclusivamente piscívora alimentación natural de los cocodrilianos.

Pérez \& Escobedo (2005), presentan las medidas de longitud y peso de 20 crías de Crocodylus acutus, recién nacidas en cautiverio en el zoocriadero piloto del Centro de Acuicultura La Tuna Carranza, Puerto Pizarro (Tumbes-Perú), en el año 2001, con un promedio de longitud total de las crías recién nacidas de $24.54 \pm 0.98 \mathrm{~cm}$ (mínimo de $21.5 \mathrm{~cm}$ y máximo de $25.5 \mathrm{~cm}$ ); mientras que el peso promedio fue de $46.13 \pm 4.28 \mathrm{~g}$ (máximo y un mínimo de 50.5 y $33.5 \mathrm{~g}$ respectivamente). Los Melanosuchus niger de esta investigación tuvieron una longitud promedio de $29.93 \mathrm{~cm}$ (rango de 29.6 a $30.8 \mathrm{~cm}$ ), y un peso promedio de $86.67 \mathrm{~g}$ (rango de 82 a 93 g). Estas variaciones indican que los neonatos de Melanosuchus niger son más grandes que de Crocodylus acutus.

Pérez (2000), en neonatos de Caiman crocodilus producidos por incubación artificial, refiere que tuvieron una longitud total que varió de 19.5 a $25.5 \mathrm{~cm}$ (promedio $22.5 \mathrm{~cm}$ ) y peso de 26.1 a $57.5 \mathrm{~g}$ (promedio de $38.2 \mathrm{~g}$ ) y después de 10 meses de cría, en tres grupos experimentales, alcanzaron una longitud de $61.5-90.5$ y $95.9 \mathrm{~cm}$ y peso promedio de $863.6 ; 2.585$ y 3.083 g. La dieta otorgada a estos animales fue para el primer grupo $70 \%$ de pescado fresco, $30 \%$ de carne pollo y mezcla de minerales. El segundo grupo $60 \%$ de pescado fresco, $40 \%$ de vísceras de res y mezcla de minerales. Para el tercer grupo la dieta fue $50 \%$ de pescado fresco $25 \%$ de vísceras de res, $25 \%$ de carne de pollo y mezcla de minerales. Por tratarse de especies diferentes, Melanosuchus niger al momento de eclosionar, tuvieron una longitud de 29.6 a $30.8 \mathrm{~cm}$ y un peso de 82 a 93 g, cifras mayores a la de Caiman crocodilus. A nivel de crecimiento nuestros caimanes negros durante el tiempo de observación crecieron de $46.22 \mathrm{~cm}$ y tuvieron un incremento de peso de $286.14 \mathrm{~g}$; resultados muy alentadores porque la alimentación suministrada a nuestros caimanes fue exclusivamente piscívora mientras que los especímenes de Caiman crocodilus fueron sometidos a dietas enriquecidas.

En Pérez (2001), en neonatos de Caiman crocodilus crocodilus incubados artificialmente, registraron una longitud promedio de $22.4 \mathrm{~cm}$ y peso de $39.1 \mathrm{~g}$, valores menores a Melanosuchus niger, que tuvieron una longitud de 29.6 a $30.8 \mathrm{~cm}$, (promedio $29.93 \mathrm{~cm}$ ) y un peso de $82 \mathrm{a} 9 \mathrm{~g}$ (promedio de $86.67 \mathrm{~g}$ ), resultados naturales por tratarse de especies diferentes. A nivel de huevos esta autora, reporta que el promedio de huevos incubados artificialmente de Caiman crocodilus crocodilus presentaron promedios de $49.1 \mathrm{~mm}$ de largo y $61.1 \mathrm{~g}$ de peso. La biometría de los huevos de Melanosuchus niger que incubamos estuvo constituida con un promedio de $119.79-121.25 \mathrm{~mm}$ de largo, $49.62-49.64 \mathrm{~mm}$ de ancho y $82.30-$ $82.99 \mathrm{~g}$ de peso, que por tratarse de especies diferentes son mayores a lo indicado por esta autora.

Staton \& Dixon (1977), señalan que los nacimientos de Caiman crocodilus crocodilus en los Llanos de Venezuela promediaron $41.5 \mathrm{~g}$ en peso (rango de 31.0 a $51.2 \mathrm{~g}$ ) y $21.49 \mathrm{~cm}$ de longitud total (rango de 19.1 a $23.8 \mathrm{~cm}$ ), cifras menores a los neonatos de Melanosuchus niger.

Medem (1981), menciona que Crocodylus intermedius "cocodrilo del Orinoco", especie en peligro crítico endémico, un crecimiento de $0.6 \pm$ $0.24 \mathrm{~mm} /$ día y $4.04 \mathrm{gr} /$ día. Nuestra investigación obtuvo un incremento diario de longitud de $0.12 \mathrm{~cm}$ $=1.2 \mathrm{~mm} /$ día y peso de $1.83 \mathrm{gr} /$ día. En longitud nuestros caimanes superan a Crocodylus intermedius más no en peso que son menores a lo indicado por el autor

Perez-Talavera (2007), reporta el crecimiento en 40 ejemplares de Crocodylus intermedius, en dos grupos, en iguales condiciones de cautiverio y alimentación, durante 11 meses y 15 días, con una dieta compuesta por $85 \%$ de carne de res, $10 \%$ de carne de pescado, $5 \%$ de huevo de gallina y mezcla de minerales y vitaminas. Los cocodrilos del grupo II alcanzaron un crecimiento promedio en longitud total $(108.5 \pm 6.89 \mathrm{~cm})$ y peso $(4.691,2 \pm 999.4 \mathrm{~g})$, los del grupo I $(103.0 \pm 6.81 \mathrm{~cm} ; 3.987 .5 \pm 978.7 \mathrm{~g})$. Nuestros valores de crecimiento en peso alcanzaron un promedio de $86.67 \mathrm{~g}$ (rango de 82 a $93 \mathrm{~g}$ ) y longitud promedio de $29.93 \mathrm{~cm}$ (rango de $29.6 \mathrm{a}$ $30.8 \mathrm{~cm}$ ), valores que se encuentran por debajo a lo reportado por esta autora por tratarse principalmente por dieta enriquecida y el nuestro una monodieta exclusivamente piscívora.

Barrios-Quiroz \& Casas-Andreu (2010), determinaron el crecimiento en 200 crías de Crocodylus moreletii "cocodrilo del pantano" en 10 lotes de 20 crías suministrándoles carne roja, pescado e hígado de pollo en diferentes proporciones. Las crías al inicio del estudio presentaron en promedio una longitud una longitud total de $261 \pm 0.17 \mathrm{~cm}$ y un peso de $44.4 \pm 1.71 \mathrm{~g}$., y el peso promedio fue de $44.4 \pm 1.17 \mathrm{~g}$. Sus resultados de crecimiento indican que la dieta $\mathrm{D}$ (carne rojahígado de pollo-pescado), mostró ( $30.3 \mathrm{~mm} / \mathrm{mes})$; la dieta $\mathrm{E}$ (carne roja) reveló $(10.3 \mathrm{~mm} / \mathrm{mes})$; la dieta $\mathrm{B}$ (pescado-hígado de pollo) tuvieron un de crecimiento $(5.5 \mathrm{~mm} / \mathrm{mes})$. Nuestra investigación a pesar de ser una monodieta en base a peces coincide con la dieta $\mathrm{D}$, y es mayor que la dieta $\mathrm{E}$ y $\mathrm{B}$. 


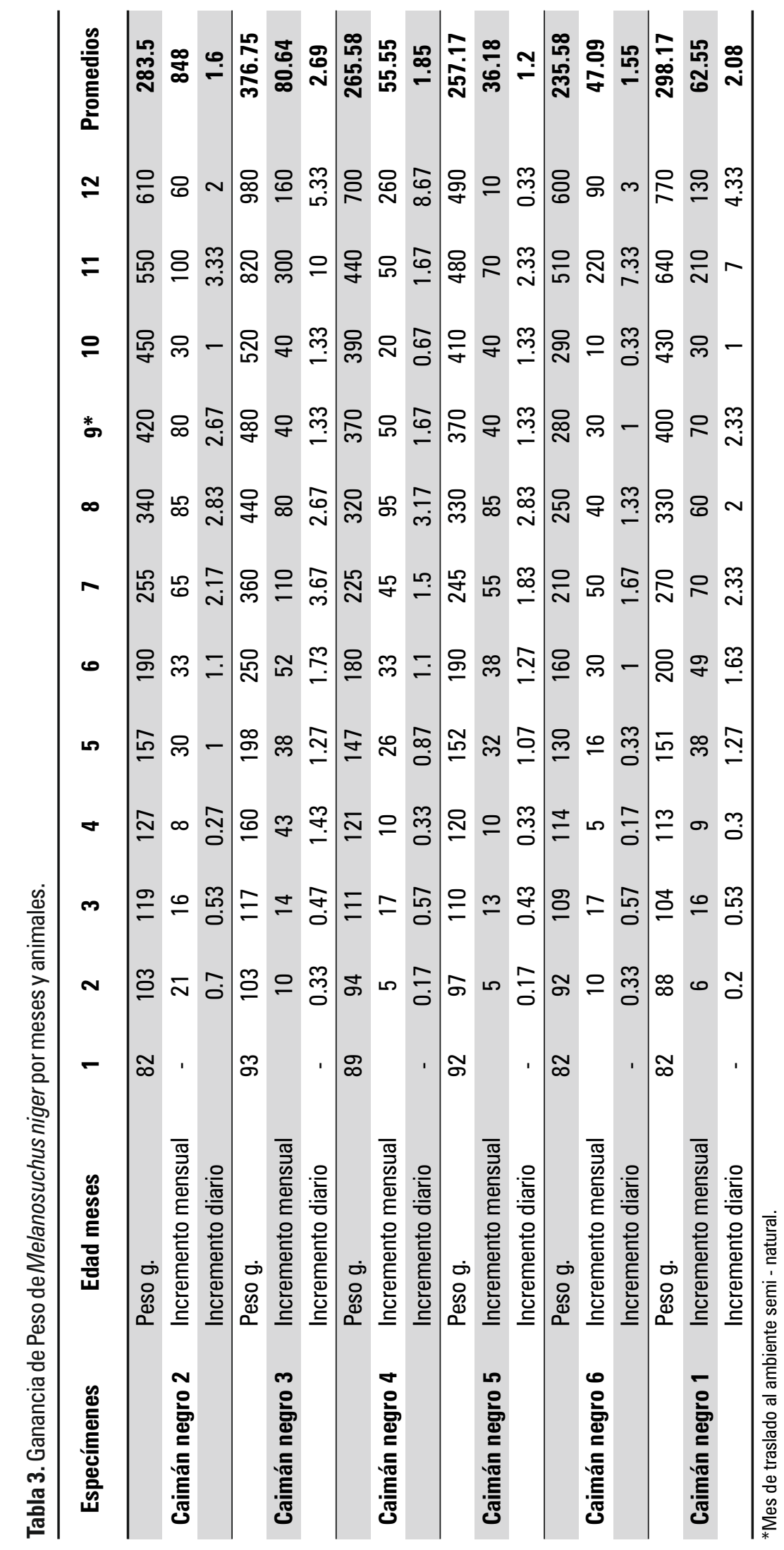




\section{CONCLUSIONES}

Los datos obtenidos en esta primera investigación resaltan las cualidades favorables al manejo que tiene Melanosuchus niger. En todo instante incrementó su longitud y peso. Preliminarmente se concluye que existe facilidad de manejo para su cría en cautiverio con una futura explotación comercial por el sistema de manejo sostenible que incluya el manejo en granjas, manejo de reproductores y caza de adultos y establecer programas de conservación para el repoblamiento en muchas áreas de nuestra Amazonía, donde sus poblaciones se encuentran seriamente disminuidas.

\section{AGRADECIMIENTO}

El presente estudio fue posible gracias al apoyo de la Universidad Nacional de la Amazonía Peruana y su Facultad de Ciencia Biológicas. Al Dr. Eckhard W. Heymann por su valioso apoyo en las sugerencias en esta investigación.

\section{BIBLIOGRAFÍA CITADA}

Albarracin, M.; Madera, R. 2011. Estudios bioecologicos del caiman negro (melanosuchus niger) en la laguna de Limoncocha, Ecuador. Facultad de Ciencias Ambientales, Universidad Internacional SEK, Quito, Ecuador. Anuario de la Universidad Internacional, SEK, 12:71-77.

Barrios-Quiroz, G.; Casas-Andreu, G. 2010. Crecimiento con diferentes dietas en crías de Crocodylus moreletii Dumeril Bibron \& Dumeril 1851 (Crocodylia: Crocodylidae) en cautiverio, Tabasco, México. Revista Latinoamericana de Conservación. 1(2): 104-111.

Brieva, C. 2002. Estado actual y perspectivas del caimán negro (Melanosuchus niger), con énfasis en la Amazonía Colombiana. Grupo de Estudio de Animales Silvestres (Boletín 2002 Geas) volumen III, Núm. 1- 6/27-33.

Cisneros, L.F. 2012. Criaturas del agua. conservación y futuro en rio Corrientes. Programa de Monitoreo Ambiental Comunitario del Río Corrientes en las actividades de manejo y conservación del lagarto negro - Pronaturaleza. Revista Viajeros Edición $\mathrm{N}^{\circ} 32$. -

Cites. 2015. Convención sobre el comercio internacional de especies amenazadas de fauna y flora silvestres. Apéndices I, II y III.

Cupul-Magaña, F.G.; Rubio-Delgado, A.; ReyesJuárez, A. 2004a. Crecimiento en talla y peso del Cocodrilo Americano (Crocodylus acutus) durante su primer año de vida. Revista Española de Herpetología. Vol.18:55-61.
Cupul-Magaña, F.G.; De Niz-Villaseñor, A.; ReyesJuárez, A.; Rubio-Delgado, A. 2004b. Historia natural del Cocodrilo Americano (crocodylus acutus) en el estero Boca Negra, Jalisco, México: anidación y crecimiento de neonatos. Ciencia y Mar, 23(VII):31-42.

Charruau, P.; Vázquez, J.R.; Villegas, A.; GonzálezCortés, H. 2010. Tasas de crecimiento del Cocodrilo Americano (Crocodylus acutus) en estado silvestre en la península de Yucatán, México. Rev. Lat. Cons., 1 (2): 63-72.

Da Silveira, R.; Campos, Z.; Thorbjarnarson, J.; Magnusson, W.E. 2013 Growth rates of black caiman (melanosuchus niger) and spectacled caiman (caiman crocodilus) from two different amazonian flooded hábitats. Amphibia-Reptilia 34: 437-449.

De la Ossa, V.J.; Vogt, R.; Rudge-Ferrara, C. 2010. Melanosuchus niger (Crocodylia: Alligatoridae) as opportunistic turtle consumer in its natural environment. Rev. Colombiana Cienc. Anim., 2(2), pp. $244-252$.

Dowling, G.; Brazaitis, P. 1966. Size and growth in captive crocodilians. International Zoo Yearbook, 6(1): 265-270.

Herron, J.C.; Emmons, L.H.; Cadle, J.1990. Observations on reproduction in the black caiman, Melanosuchus niger. Journal of Herpetology, 24(3): 316-317.

Herron, J.C. 1991. growth rates of black caiman Melanosuchus niger and Caiman crocodilus \& and the recruitment, of breeders in hunted caiman populations. Biological Conservation, 55:103-113.

Horna, V.; Zimmermann, R.; Cintra, R.; Vásquez, P.; Horna, J. 2003. Feeding ecology of the black caiman (Melanosuchus niger) in Manu National Park, Peru. Lyonia 4(1): 65-72.

Jacobsen, T.; Kushlan, J.A.1989. Growth dynamics in the American alligator (Alligator mississippiensis). Journal of Zoology (London) 219:309-328.

Jiménez-Alonso, G.; Afanador-Tellez, G.; ViorelloCifuentes, J. 2006. Evaluación de la inclusión de harina integral de carne de gallina de desecho en alimentos completos balanceados para neonatos de babilla (Caiman crocodilus fuscus; cope, 1868) y su efecto sobre el crecimiento y la productividad en cautiverio. Mem. Conf. Interna Med. Aprovech. Fauna Silv. Exót. Conv. 2: 1.

Magnusson, w.; Sanaiotti, T. 1995. Growth of caiman crocodilus crocodilus in central amazonia, Brazil. Copei., 2: 498-501. 
Medem, F. 1981. Los Crocodylia de Sur América. Volumen 1. Los Crocodylia de Colombia. Conciencias. Bogotá, Colombia.356 pp

Meraz, J.; Montoya, J.A.; Ávila, E.; Reyes, L. 2008. Monitoreo del crecimiento del Cocodrilo Americano crocodylus acutus, durante su primer año de vida en condiciones de cautiverio. Hidrobiológica, 18 (2): 125-136.

Ministerio de Agricultura. 2014. Decreto Supremo $\mathrm{N}^{\circ}$ 004-2014-Minagri. Actualización de la lista de clasificación y categorización de las especies amenazadas de fauna silvestre legalmente protegidas.

Neira, J.A.1994. Reproducción de Melanosuchus niger spix, 1825, caimán negro en cautiverio. Trianea. Num.5. ISSN: 0121-55-82. pp: 109121.

Pask, D. 2005. The abundance of three caiman species (Caiman crocodilus, Paleosuchus trigonatus and Melanosuchus niger) along the río Javari, Peru/Brazil. Unpublished BSc thesis.

Pérez, A.T. 2000. Crecimiento del Caiman crocodilus en cautiverio. Interciencia, Revista de Ciencia y Tecnología, diciembre, año/vol.25, número 009. Asociación Interciencia Caracas Venezuela. pp. 442-446.

Pérez, A.T. 2001. Incubación artificial de huevos de baba (Caiman crocodilus crocodilus). Zootecnia Tropical, 19(2): 154-164.

Pérez A.T.; Velasco, Á. 2002. Evaluación del crecimiento de Crocodylus intermedius con una dieta experimental. Bol. Centro lnvest. Biol. 36(2) 125-135.

Pérez, A.T. 2007. Efecto del manejo por captura y medición en el crecimiento del Caiman del Orinoco (Crocodylus intermedius) en cautiverio. Boletín del Centro de Investigaciones Biológicas, 41(2): 208-214.

Pérez, A.; Rodríguez, J.C. 2005. Influencia de la temperatura del aire y del agua en el crecimiento de Crocodylus intermedius en dos condiciones de cautiverio. Boletín del Centro de Investigaciones Biológicas, 39(1): 15-26.

Pérez, O.; Escobedo, A.H. 2005. Observaciones biométricas de Crocodylus acutus (Cuvier, 1807) recién nacidos en cautiverio, Tumbes, Perú. Rev., Perú. Biol. 12(1): 171-172.

Pérez, O: Escobedo-Galvan, A. 2007. Crecimiento en cautiverio de Crocodylus acutus (Cuvier, 1807), en Tumbes, Perú. Revista Peruana de biología, 14(2): 221-223.

Pérez, M.; Naranjo, C.; Reyes, B.; Veja, I. 2009. Influencia de dos tipos de dietas sobre la talla y el peso corporal en neonatos de Crocodylus acutus Cuvier, 1807 (Crocodylidae: Crocodylia) del zoocriadero de manzanillo, Cuba. Acta Zoológica Mexicana (nueva serie), 25(1): 151160.

Piedra, C.L.; Bolaños, J.R.; Sánchez, R.J. 19961997. Evaluación del crecimiento de neonatos de Crocodylus acutus (Crocodilia: Crocodylidae) en cautiverio. Rev. Biol. Trop., 44(3) /45(1): 289293.

Plotkin, M.J.; Medem, F.; Mittermeier, R.A.; Constable, I.D. 1983. Distribution and conservation of the blanck caiman (Melanosuchus niger). Advances in Herpetology and Evolutionary Biology, $695-705$ pp.

Rebelo, G.H.; Magnusson, W. E. 1983. An analysis of the effect of hunting on Caiman Crocodilus and Melanosuchus niger based on the sizes of confiscated skins. Biol. Conserv., 26, 95-104.

Rodríguez, M.; Miguel, A. 1988. Anotaciones sobre el crecimiento de neonatos y juveniles de Caiman crocodilus fuscus (cope, 1868) Crocodilia - Alligatoridae). Trianea (Act. Cien.Tecn. INDERENA), 1:71-77.

Rodríguez M. Miguel A. 1989. Tres modelos Crocodylus fuscus (Cope, 1968). (Crocodilia: Alligatoridae) en cautiverio. TRIANEA (Act. Cient. Tecn. Inderena) 3:61-66.

Rodríguez, E.; Rodríguez, M.1989. Evaluación del crecimiento y levante de neonatos y juveniles de Crocodylus acutus Cuvier 1807, (Crocodilia: Crocodylidae) durante 1987-1988 en el centro experimental de fauna silvestre de San Marcos (sucre). Trianea (Act. Cient. Tecno. INDERENA), 3:53-60.

Rodríguez, M.; Rodríguez, A. 1991. La frecuencia y la tasa de alimentación como claves para el crecimiento de neonatos y juveniles de Crocodylus acutus, Cuvier 1807. (Crocodilia: Crocodylidae). TRIANEA (Act. Cient. Teca. INDERENA), 4: 497-504.

Rueda-Almonacid, J. V., J. L. Carr, R. A. Mittermeier, J. V. Rodriguez-Mahecha, R.B. Mast, R. C. Vogt, A. G. J. Rhodin, J. de La Ossa, J. N. Rueda y C. G. Mittermeier.2007. Las tortugas y los crocodilidos de los países andinos del trópico. Serie de guías tropicales de campo No 6. Conservación Internacional. Editorial Panamericana, Formas e Impresos, Bogotá, Colombia.

Staton, M.A.; Dixon, J.R. 1977. The breeding biology of the spectacled caiman, Caiman crocodilus crocodilus, from Venezuelan llanos. U.S.A. Fish and Wildl. Service Wildl Res. Rep., $5: 21$. 
Tejado, C. 2012. Abundancia de Melanosuchus niger, Spix, 1825, Paleosuchus trigonatus, Schneider 1801 y Caiman crocodilus L., 1758 en el cauce medio del río Mazán (Amazonas, Perú). Munibe (Ciencias Naturales-Natur Zientziak) No 60 Donostia / San Sebastián ISSN 02147688

Thorbjarnarson, J.1989. Ecology of the American crocodile, Crocodylus acutus. In Crocodriles international union for conservation of nature and natural resources. (ed.). Crocodiles Their ecology, management, and conservation. New Series. Gland, Switzerland, 228-258 pp.

Thorbjarnarson, J.B. 2010. Black caiman Melanosuchus niger. pp: 29-39 in Crocodiles. Status Survey and Conservation Action Plan. Third Edition, ed. by S.C. Manolis and C. Stevenson. Crocodile Specialist Group: Darwin.
Vieira, L.G.; Lima, F.C.; Santos, A.L.Q.; Mendoça, S.H.S.T.; Moura, L.R.; Iasbeck, J.R.; Sebben, A. 2011. Description of embryonic stages in Melanosuchus niger (Spix, 1825) (Crocodylia: Alligatoridae). J. Morphol. Sci., 28:(1): 11-22.

Villamarín, J.F.J. 2006. Anidación y patrones de uso de hábitat del caimán negro, Melanosuchus niger (Crocodilia: Alligatoridae), en dos localidades de la Amazonía Ecuatoriana. Disertación previa a la obtención del título de Licenciado en Ciencias Biológicas. Pontificia Universidad Católica del Ecuador Facultad de Ciencias Exactas y Naturales Escuela de Ciencias Biológicas. Quito.108 p.

Recibido: 1 de octubre del 2015

Aceptado para publicación: 30 de noviembre del 2015 